J. Gynäkol. Endokrinol. AT 2019 • 29:67-68 https://doi.org/10.1007/s41974-019-00110-1 Online publiziert: 1. Oktober 2019 (c) Springer-Verlag GmbH Austria, ein Teil von Springer Nature 2019

\title{
Franz Fischl
}

Wien, Österreich

\section{Editorial}

Liebe Leserinnen und Leser!

Die Sommerpause neigt sich dem Ende zu. Nach dem bisher zweitheißesten Sommer seit den meteorologischen Aufzeichnungen, der immer wieder von heftigen lokalen Unwettern unterbrochen wurde, melden wir uns mit der Herbstausgabe voller Tatendrang zurück. Der heißdiskutierte Klimawandel wird auch vor der Medizin nicht Halt machen und wir müssen uns sehr bald damit intensiv auseinandersetzen, was bedeutet die Klimaerwärmung für uns in Europa, welche Erkrankungen können dadurch bei uns regelmäßig auftauchen? Wir werden uns mit dieser Problematik auch bald in unserem Journal beschäftigen. Wir hoffen, Sie hatten wunderschöne erholsame Urlaubstage und erwarten nun mit Spannung und Freude diese neue Ausgabe.

Katharina Feil et al. präsentieren die neuen ESHRE-Leitlinien und aktuelle Studien die prämature Ovarialinsuffizienz betreffend. Der frühe Verlust der Ovarialfunktion (vor dem 40. Lebensjahr) wird prämature Ovarialinsuffizienz (POI) genannt und tritt in rund 1-2\% auf. Die Ursachen können vielfältig sein, der größte Anteil ist jedoch idiopathisch. Die Schwangerschaftsrate ist sehr niedrig und meistens ist die Erfüllung des Kinderwunsches nur durch eine Eizellspende möglich. Die Autorinnen zitieren auch neuere Studien, die bei diesem Krankheitsbild auf ein erhöhtes kardiovaskuläres Risiko, ein erhöhtes Auftreten von Osteoporose und andere Östrogenmangelsymptome und letztendlich sogar auf eine reduzierte Lebenserwartung hinweisen.

Elisabeth Janschek bringt in ihrer Arbeit einen aktuellen Überblick über die Adenomyose. In diesem Artikel gibt die Autorin rezente Einblicke in die Patho- genese, in die aktuellen diagnostischen Verfahren und geht zuletzt auf mögliche organerhaltende Therapieoptionen ein.

Sonja Kickmaier und W. Marktl geben in ihrer Arbeit „Studien - was gibt es Neues?" Einblick in die modernen Anforderungen von Studien in unserer Zeit. Die moderne Forschung und die rasante Entwicklung der Wissenschaft verlangen die Etablierung einer neuen Studiengeneration. Die Autoren beschreiben die modernen Möglichkeiten einzelner Methoden.

Im News-Screen Assistierte Reproduktion stellt uns $M$. Feichtinger 2 interessante Originalarbeiten vor, die sich mit dem Erfolg von Sterilitätsbehandlungen bei regelmäßigem Kaffee- und Alkoholkonsum von Frauen mit Kinderwunsch auseinandersetzen. Interessanterweise konnten keine Unterschiede in den Schwangerschafts- und Lebendgeburtenraten zwischen kein Koffein- bzw. Alkoholgenuss, nur mäßigem und relativ starkem Koffein- bzw. Alkoholgenuss während der Sterilitätsbehandlung (IVF/ ICSI) gefunden werden. Da sehr schnelle Lebensstiländerungen während einer Kinderwunschbehandlung zusätzlichen Stress hervorrufen können, zeigen diese Studien, dass gerade in dieser Phase eine Lebensstiländerung für bessere Ergebnisse nicht unbedingt nötig ist.

Im News Screen Menopause berichtet $P$. Frigo über 4 Originalarbeiten, die sich im Wesentlichen mit Schlafstörungen in der Menopause auseinandersetzen. Nachdem Schlafstörungen in der Menopause zu den häufigsten Beschwerden zählen und verschiedenste Ursachen haben können, gehen diese Studien die Problematik von ganz verschiedenen Ansätzen an. Es ist evident, dass die individuellen Ursachen von Schlafproblemen nach deren Evaluierung entspre- 


\section{Editorial}

chend abgeklärt und untersucht werden müssen, um entsprechende Therapieoptionen individuell anzuwenden.

Ch. Brezinka bringt uns in seinen Tipps und Tricks im Gyn-Ultraschall in seinem Beitrag „Die Gestationsalterbestimmung mit Ultraschall“ die Möglichkeiten einer genauen Geburtsterminbestimmung, gegenüber den oft nur ungenauen Berechnungen, näher und gibt wie immer wertvolle Tipps für die Praxis.

In der Mädchensprechstunde setzt sich Kollegin Iris Holzer, unsere neue Chefredakteurin, mit dem Thema der primären Amenorrhoe auseinander. Es ist wichtig, nicht allzu lange mit der Abklärung und wenn nötig mit der entsprechenden Therapie zu warten, wenn die Menarche nicht bis nach dem 15. Lebensalter eintritt. Eine entsprechende Abklärung, bestehend aus klinischer Beurteilung der Körperentwicklung (Pubertätsentwicklung), Ultraschall zur Diagnostik von Uterus und Ovarien sowie ein Hormonstatus, soll differentialdiagnostisch mögliche Ursachen aufzeigen.

In Menopause heute und morgen zeigt uns Kollegin Iris Holzer in „Einfluss einer Hormonersatztherapie auf das vaginale Mikrobiom" an Hand von neueren Publikationen, wie durch den Östrogenmangel in der Menopause sich auch das vaginale Mikrobiom negativ verändert. Durch eine entsprechende Östrogentherapie kommt es $\mathrm{zu}$ einer deutlichen $\mathrm{Zu}$ nahme von Laktobazillen, wodurch eine deutliche Besserung der vaginalen Symptome wie Scheidentrockenheit, Juckreiz und Atrophie erreicht wird.

Pharma-News und ein Terminkalender runden den Inhalt dieser Ausgabe in gewohnter Weise ab.

Der Verlag und ich wünschen Ihnen für den nun nahenden Herbst wieder eine interessante und spannende Lektüre mit vielen Anregungen für die Praxis. Gerne nehmen wir auch Wünsche und Anregungen von Ihnen für das Journal auf.

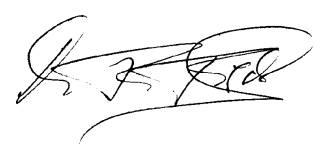

Franz Fischl

Herausgeber

\section{Korrespondenzadresse}

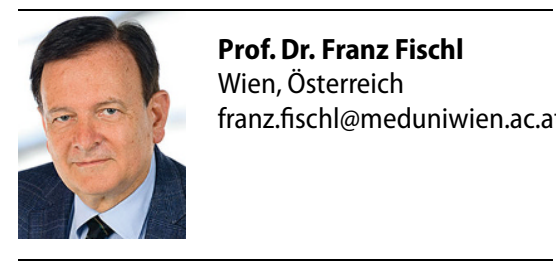

Interessenkonflikt. F. Fischl gibt an, dass kein Interessenkonflikt besteht.

Hinweis des Verlags. Der Verlag bleibt in Hinblick auf geografische Zuordnungen und Gebietsbezeichnungen in veröffentlichten Karten und Institutsadressen neutral. 